\title{
Metastatic lung adenocarcinoma to the bladder: A case report
}

\author{
HAI-JUN YE ${ }^{1 *}$, JIAN MA $^{2 *}$, YING-JIE LIU ${ }^{1}$, XIAO-FEI YE ${ }^{1}$, LI-WANG ZHANG ${ }^{2}$ and JIN-GE LI $^{3}$ \\ ${ }^{1}$ Department of Pathology, People's Liberation Army 205 Hospital, Jinzhou, Liaoning 121000; \\ Departments of ${ }^{2}$ Research Service, and ${ }^{3}$ Infectious Disease, Tangdu Hospital, \\ The Fourth Military Medical University, Xi'an, Shaanxi 710038, P.R. China
}

Received August 30, 2014; Accepted June 2, 2015

DOI: $10.3892 / \mathrm{ol} .2015 .3445$

\begin{abstract}
Urothelial cancer is the most frequently diagnosed type of malignant tumor in the bladder, of which primary adenocarcinoma accounts for a small percentage. Secondary malignancies, in particular metastatic adenocarcinoma from the lung, are exceedingly rare, with only six cases previously reported in the literature. The present study describes the case of a 71-year-old Chinese male patient with known lung cancer for $>2$ years, who was diagnosed with metastatic adenocarcinoma to the bladder. The histopathological characteristics and immunohistochemical features of the patient are reported. It was proposed that pathologists should consider the possibility of metastatic adenocarcinoma from the lung, rather than assume a diagnosis of primary adenocarcinoma of the bladder or direct invasion of adenocarcinoma from the surrounding organs. Furthermore, it is essential to determine the medical history of each patient and observe the immunohistochemical features of all tumors prior to diagnosis.
\end{abstract}

\section{Introduction}

Bladder cancer is the fifth most common type of malignant tumor. Among bladder malignancies, urothelial carcinomas are the most frequent histological type, of which adenocarcinoma, squamous cell cancer and small-cell cancer account for small percentages $(1,2)$. Primary adenocarcinomas were reported to account for $0.5-2 \%$ of primary bladder epithelial malignancies; these malignancies most commonly arise in the urachus (3).

Correspondence to: Dr Li-Wang Zhang, Department of Research Service, Tangdu Hospital, The Fourth Military Medical University, 569 Xinsi Road, Xi'an, Shaanxi 710038, P.R. China

E-mail: zhanglw@fmmu.edu.cn

Professor Jin-Ge Li, Department of Infectious Disease, Tangdu Hospital, The Fourth Military Medical University, 569 Xinsi Road, Xi'an, Shaanxi 710038, P.R. China

E-mail: jingeli@126.com

${ }^{*}$ Contributed equally

Key words: lung adenocarcinoma, bladder, metastatic, thyroid transcription factor-1
In addition, secondary adenocarcinomas may also occur as a result of direct invasion from the surrounding organs, including prostate, colorectal and cervical sites (3). Furthermore, metastatic spread may occur via lymphogenous or hematogenous routes (4). However, the bladder is not a favored site for tumor metastases; in particular, adenocarcinoma from the lung is exceptionally rare (5). The present study describes a case of metastatic adenocarcinoma from the lung to the bladder and reports the histopathological characteristics and immunohistochemical features of the patient. The aim of the present study was to emphasize the importance of considering of metastatic adenocarcinoma from the lung as a possible diagnosis, in addition to primary adenocarcinoma and direct invasion of adenocarcinoma from the immediate surrounding organs.

\section{Case report}

A 71-year-old Chinese male with known lung cancer for $>2$ years was referred to the People's Liberation Army 205 Hospital (Jinzhou, China) due to a gross hematuria, which had persisted for 5 days. This initial diagnosis of lung adenocarcinoma occurred $>2$ years previously, when the patient was admitted to a local hospital due to a cough and expectoration. Following the initial admission to hospital, a thoracic computed tomography (CT) revealed a $3 \times 2 \times 2 \mathrm{~cm}$ mass in the lower lobe of the patient's left lung; consequently, a needle biopsy was performed and the patient was diagnosed with adenocarcinoma of the lung (histological sections were not available for review). The patient elected not to undergo any therapy, including surgical resection, chemical therapy or radiotherapy. On admission to the People's Liberation Army 205 Hospital due to gross hematuria, ultrasonography of the pelvic cavity was performed, which revealed a solid occupying lesion, $0.6 \times 0.4 \times 0.4 \mathrm{~cm}$ in size, within the right lateral wall of the bladder. Consequently, a transurethral resection of a bladder tumor was performed. During this procedure, the tumor was identified to be pedunculated and conch-shaped with a smooth surface. Histopathological analysis of the resected tumor revealed that the tumor cells grew diffusely and were located beneath the intact urothelial epithelium, without bladder muscle invasion; in addition, mucus was identified in certain tumor cells, as determined following hematoxylin and eosin staining (Fig. 1A). Immunohistochemically, the tumor cells stained positively for cytokeratin (CK)7, thyroid transcription factor-1 (TTF-1) and Napsin A (Fig. 1B-D, respectively); however, the tumor cells were negative for CK20, 
A

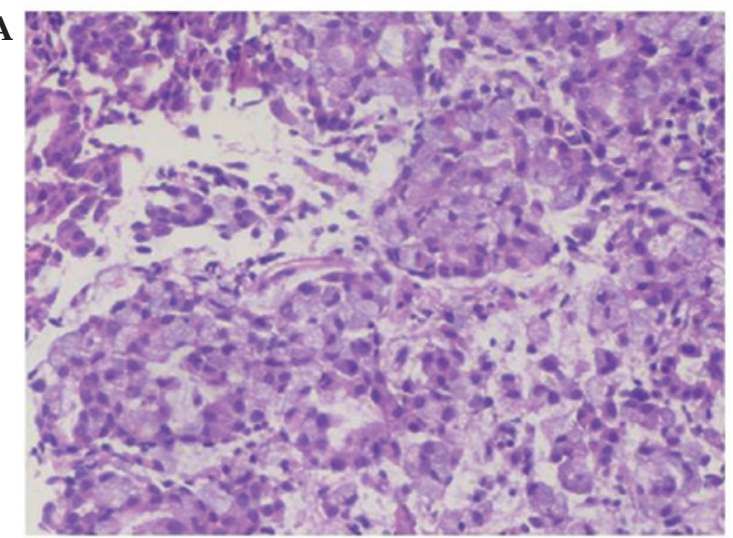

C

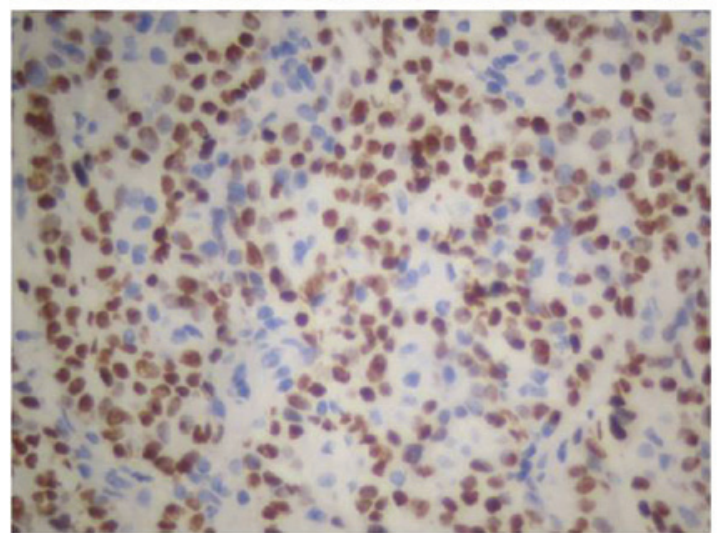

B

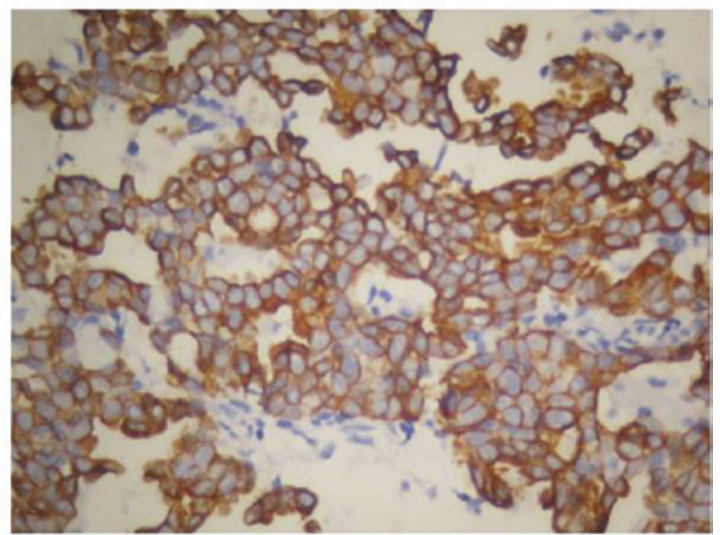

D

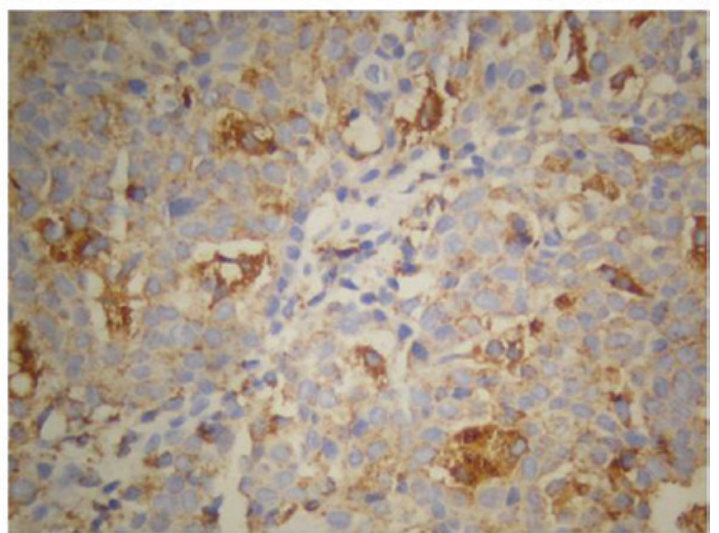

Figure 1. Histopathological analysis of tumor cells. Tumor cells grew diffusely and were located beneath the intact urothelial epithelium, without bladder muscle invasion. (A) Hematoxylin and eosin staining identified mucus present in certain tumor cells. Immunohistochemical analysis demonstrated that the tumor cells were strongly positive for (B) cytokeratin 7, (C) thyroid transcription factor-1 and (D) NapsinA.

prostate-specific antigen (PSA), P504S, prostatic acid phosphatase (PAP) and caudel-type homeobox (CDX)2. Combining the histopathological and immunohistochemical features with the patient's clinical history, it was considered that the tumor was a metastatic adenocarcinoma from the lung; however, the primary tumor slides were not available for review. This conclusion was reached as adenocarcinoma from the prostate would usually express PSA, PAP and P504S, while adenocarcinoma from the gastrointestinal tract are often positive for CK20 and CDX2. Written informed consent was obtained from the patient prior to publication of the present study.

\section{Discussion}

Cancer metastases to the bladder from adjacent sites, including prostate, colorectal and cervical sites, are common; whereas metastases from stomach, skin, breast and lung primary tumors are less common (4). Metastatic adenocarcinoma from the lung to the bladder is rare and only six cases have been reported in the literature since 1997 (4-9). Furthermore, in a retrospective study from 282 cases of secondary bladder neoplasms, only one case $(0.4 \%)$ originating from lung adenocarcinoma was identified (5).

Among the six cases reported in the literature, four cases presented clinical data, including age, gender and clinical symptoms (4,7-9). The age range of the subjects was 40-81 years, with a mean age of 66 years. Three of the patients were male and one was female. Clinically, hematuria and pelvic pain are often the presenting symptoms in patients with secondary bladder cancer (10). Out of these previous studies, two patients presented with gross hematuria, one presented with pain and bilateral hydronephrosis, and one was initially referred to the neurosurgical clinic for left carpal tunnel syndrome. However, both patients were confirmed to have a history of primary lung cancer following careful physical examination $(8,9)$. In the current case, the patient presented with a gross hematuria $>2$ years following diagnosis of the primary lung adenocarcinoma. Ultrasonography of the pelvic cavity revealed a solid occupying lesion, $0.6 \times 0.4 \times 0.4 \mathrm{~cm}$ in size, within the right lateral wall of the bladder. Histopathological analysis identified the lesion as an adenocarcinoma. Primary adenocarcinoma of the bladder and secondary adenocarcinoma from its adjacent organs should be ruled out based on immunohistochemical studies, which may be used to determine the origin of the primary tumor from uncommon sites, such as colorectal, breast or lung. Positive staining for TTF-1, CK7 and Napsin A have been demonstrated to be reliable markers for the identification of lung adenocarcinoma $(2,11)$. In the present case report, the tumor cells stained positive for TTF-1, CK7 and Napsin A. In addition, the tumor cells were negative for CK20, PSA, P504S, PAP and CDX2; thus, adenocarcinomas from the prostate, rectum and stomach may be excluded.

In conclusion, it is proposed that in cases comparable to the current study, pathologists should consider the possibility of metastatic adenocarcinoma from the lung, rather than primary adenocarcinoma of the bladder or direct invasion of adenocarcinoma from the surrounding organs. Furthermore, 
it is essential to determine the medical history of each patient and observe the immunohistochemical features of all tumors prior to diagnosis.

\section{References}

1. Howlader N, Noone AM, Krapcho M, et al (eds): SEER Cancer Statistics Review, 1975-2009 (Vintage 2009 Populations) National Cancer Institute, Bethesda, MD, 2012.

2. Wasco MJ, Daignault S, Zhang Y, et al: Urothelial carcinoma with divergent histologic differentiation (Mixed Histologic Features) predicts the presence of locally advanced bladder cancer when detected at transurethral resection. Urology 70: 69-74, 2007.

3. Thomas DG, Ward AM and Williams JL: A study of 52 cases of adenocarcinoma of the bladder. Br J Urol 43: 4-15, 1971.

4. Modh RA, Corbyons KA and Yeung LL: A rare metastasis to the bladder. Case Rep Urol 2013: 789039, 2013.

5. Bates AW and Baithun SI: Secondary neoplasms of the bladder are histological mimics of nontransitional cell primary tumours: Clinicopathological and histological features of 282 cases. Histopathology 36: 32-40, 2000.
6. Martín-Marquina Aspiunza A, Díez-Caballero Alonso F, Rodríguez-Rubio Cortadellas FI, Díez Fernández L, Abad Vivas-Pérez JI, et al: Bladder metastasis of lung adenocarcinoma. Actas Urol Esp 21: 406-408, 1997 (In Spanish).

7. Sakhri L, Mennecier B, Jacqmin D, Di Marco A, Schumacher C, Chenard MP, Bergmann E and Quoix E: Atypical metastatic site of lung adenocarcinoma. Rev Pneumol Clin 67: 375-379, 2011 (In French)

8. Karle W, Barazani Y and Tareen B: A rare case of metastatic lung cancer to the bladder. Can Urol Assoc J 6: E147-E149, 2012.

9. Shirakawa H, Kozakai N, Sawafuji M, Sugiura H and Hara S: Urinary bladder metastasis originating from lung adenocarcinoma: A case definitively diagnosed by immunohistochemistry. Urol J 9: 530-532, 2012.

10. Acino SM and Hampel N: Renal cell carcinoma presenting with gross haematuria from a solitary bladder metastasis. Eur Urol 15: 294-296, 1988.

11. Turner BM, Cagle PT, Sainz IM, et al: Napsin A, a new marker for lung adenocarcinoma, is complementary and more sensitive and specific than thyroid transcription factor 1 in the differential diagnosis of primary pulmonary carcinoma: evaluation of 1674 cases by tissue microarray. Arch Pathol Lab Med 136: 163-171, 2012. 